\title{
Extreme ultraviolet probing of nonequilibrium dynamics in high energy density germanium
}

\author{
E. Principi,${ }^{1, *}$ E. Giangrisostomi, ${ }^{1,2}$ R. Mincigrucci, ${ }^{1}$ M. Beye,${ }^{3}$ G. Kurdi, ${ }^{1}$ R. Cucini, ${ }^{1}$ \\ A. Gessini, ${ }^{1}$ F. Bencivenga, ${ }^{1}$ and C. Masciovecchio ${ }^{1}$ \\ ${ }^{1}$ Elettra-Sincrotrone Trieste S.C.p.A., S.S. 14 km 163.5, 34149 Basovizza (TS), Italy \\ ${ }^{2}$ Helmholtz-Zentrum Berlin für Materialien und Energie GmbH, Albert-Einstein-Straße 15, 12489 Berlin, Germany \\ ${ }^{3}$ DESY (FS-FLASH) Notkestrasse 85, D-22607 Hamburg, Germany
}

(Received 27 November 2017; published 29 May 2018)

\begin{abstract}
Intense femtosecond infrared laser pulses induce a nonequilibrium between thousands of Kelvin hot valence electrons and room-temperature ions in a germanium sample foil. The evolution of this exotic state of matter is monitored with time-resolved extreme ultraviolet absorption spectroscopy across the Ge $M_{2,3}$ edge $(\simeq 30 \mathrm{eV})$ using the FERMI free-electron laser. We analyze two distinct regimes in the ultrafast dynamics in laser-excited Ge: First, on a subpicosecond time scale, the electron energy distribution thermalizes to an extreme temperature unreachable in equilibrium solid germanium; then, during the following picoseconds, the lattice reacts strongly altering the electronic structure and resulting in melting to a metallic state alongside a breakdown of the local atomic order. Data analysis, based on a hybrid approach including both numerical and analytical calculations, provides an estimation of the electron and ion temperatures, the electron density of states, the carrier-phonon relaxation time, as well as the carrier density and lattice heat capacity under those extreme nonequilibrium conditions. Related structural anomalies, such as the occurrence of a transient low-density liquid phase and the possible drop in lattice heat capacity are discussed.
\end{abstract}

DOI: 10.1103/PhysRevB.97.174107

\section{INTRODUCTION}

The availability of intense femtosecond lasers have revolutionised the experimental study of materials under high energy density $\left[(\mathrm{HED}) \gtrsim 10^{4} \mathrm{~J} \mathrm{~cm}^{-3}\right]$ conditions, giving access to unexplored states of matter [1], albeit ubiquitously present in extraterrestrial environments. With subpicoseconds pulses, the excitation occurs faster than the typical time scale for electron-phonon coupling. The excited electronic system only equilibrates with the lattice on a longer time scale. This results in a transient exotic nonequilibrium state, characterized by a hot valence electron subsystem and a room-temperature ion lattice $[2,3]$. Within the following few picoseconds, valence electrons and ions thermalize, possibly leading to an unusual state of matter at the boundary between ideal plasmas and the condensed phase, called warm dense matter [4]. This rather unexplored state of matter lacks sophisticated theoretical descriptions and calls for new experimental findings to gain a fundamental understanding with direct implications also for HED applied research, e.g., for inertial confinement fusion [5].

Tetrahedral diamondlike materials (e.g., silicon or germanium) exhibit peculiar dynamics under HED conditions and are therefore excellent sample candidates for experiments with intense pulsed lasers. Ultrafast x-ray probing of the nonequilibrium state provides unprecedented insights into the picosecond dynamics of the ion and electron subsystems. For example, Siders et al. [6], Sokolowski-Tinten et al. [7], and Sokolowski-Tinten and von der Linde [8] pioneered timeresolved x-ray diffraction experiments on $\mathrm{Ge}$ shedding light

\footnotetext{
*Corresponding author: emiliano.principi@elettra.eu
}

on a laser-induced subpicosecond breakdown of the atomic long-range order, termed "nonthermal melting." Beye et al. [9] performed time-resolved soft x-ray emission spectroscopy on Si thus revealing the occurrence of a semimetallic low-density liquid (LDL) phase in Si after about 1 ps from laser excitation. More recently, Zürch et al. [10] used time-resolved x-ray absorption spectroscopy (trXAS) to monitor the subpicosecond carriers dynamics in nanocrystalline Ge using a laser-based high harmonic generation source although not in the HED regime.

Here, we show results from trXAS at the Ge $M_{4,5}$ edge to probe the electronic structure evolution under HED conditions with sub-100-fs resolution. This time resolution, suitable for monitoring ultrafast changes in the electron density of states in condensed materials, has been achieved avoiding the use of sophisticated streak cameras [11,12] and carrying out the spectroscopy by scanning the photon energy. Using the high brilliance of the FERMI free-electron laser (FEL) in Trieste (Italy) together with the essentially jitter-free timing [13] and accurate spectral stability $\left(\lesssim 10^{-3}\right.$ without additional monochromatization), the required number of shots for statistically meaningful results can be limited to five to ten per data point, acquired in less than a minute. Since every single shot is destructive to the sample, the measurements have been conducted by raster scanning the sample.

\section{EXPERIMENTAL DETAILS}

The experiment was carried out at the EIS-TIMEX beamline [14]. The sample consisted of a self-standing microcrystalline Ge foil, provided by the Lebow Company, of nominal thickness $d=80 \mathrm{~nm}$, chosen to optimize the absorption level of the 


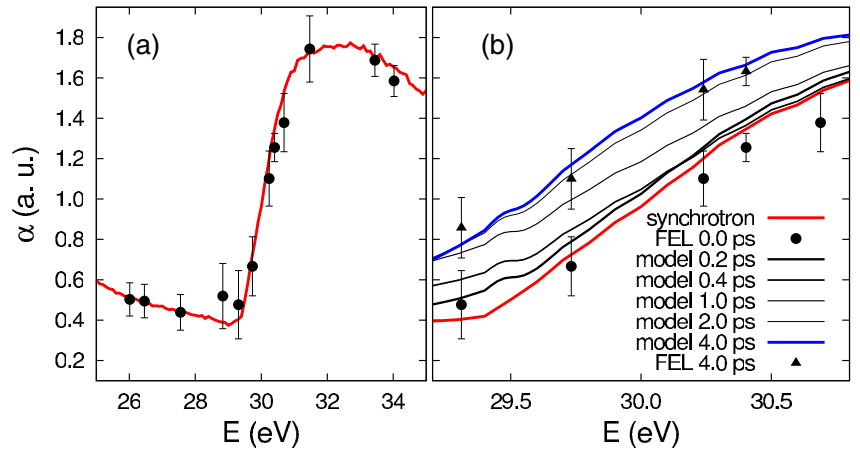

FIG. 1. (a) Absorption spectra of Ge across the $M_{4,5}$ edge obtained at the synchrotron (solid curve) and at the FEL (FERMI), EIS-TIMEX beamline (circles). (b) Time-resolved dynamics of the $M_{4,5}$ edge as obtained from optical laser-pump/FEL-probe measurements and theoretical calculations (see the text and Fig. 2).

extreme ultraviolet (EUV) probe across the Ge $M_{4,5}$ edge. The sample was excited by a fraction of the laser that seeds the FEL process. We used a wavelength of $780 \mathrm{~nm}$ with a pulse duration of $90 \mathrm{fs}$ impinging nearly normal onto the sample. The diameters of the FEL probe and laser pump spots at the sample were 30 and $170 \mu \mathrm{m}$, respectively. The laser pump fluence was set at about $45 \mathrm{~mJ} \mathrm{~cm}^{-2}$ corresponding to an average energy density of about $0.5 \times 10^{4} \mathrm{~J} \mathrm{~cm}^{-3}(\approx 1 \mathrm{MJ} / \mathrm{kg}$ considering typical Ge crystal density). The largest fraction of the optical energy is expected to be concentrated $40-50 \mathrm{~nm}$ beneath the surface [7] thus inducing isochoric HED conditions in more than $50 \%$ of the sample. The attained energy density can bring Ge up to temperatures of a few $\mathrm{eV}$ and pressures in the GPa regime.

The absorption measurements were carried out in transmission geometry by tuning the FEL wavelength to the desired values. Every pump-probe event was preceded by a sequence of three probe shots to estimate the local unperturbed sample absorption. After the pump-probe pulse pair, the sample was moved to a fresh position. The probe beam intensity upstream of the sample was measured by an ionization chamber. The portion of the probe EUV beam transmitted by the sample impinged on a cerium-doped yttrium aluminum garnet (YAG) crystal whose fluorescence was measured with a photodiode (UVG100 by IRD). The YAG crystal was coated by aluminum to block undesired background from the seed and pump lasers. We demonstrate the quality of this setup by comparing a reference Ge $M_{4,5}$ edge absorption spectrum recorded under ambient conditions [Fig. 1(a)] at the Elettra synchrotron facility (BEAR beamline) with the spectrum resulting from absorption of strongly attenuated FEL pulses measured at EIS-TIMEX.

\section{RESULTS AND DISCUSSION}

In Fig. 1(b), we show the near-edge absorption spectrum after exposure to the laser pump as a function of time. One can note a redshift of the $M_{4,5}$ edge ( $\Delta E \approx 0.5 \mathrm{eV}$ ) that begins after a few hundreds of femtoseconds and is completed in a few picoseconds. A similar observation, but without temporal resolution, is reported, for example, on the $L_{1}$ edge of laser-

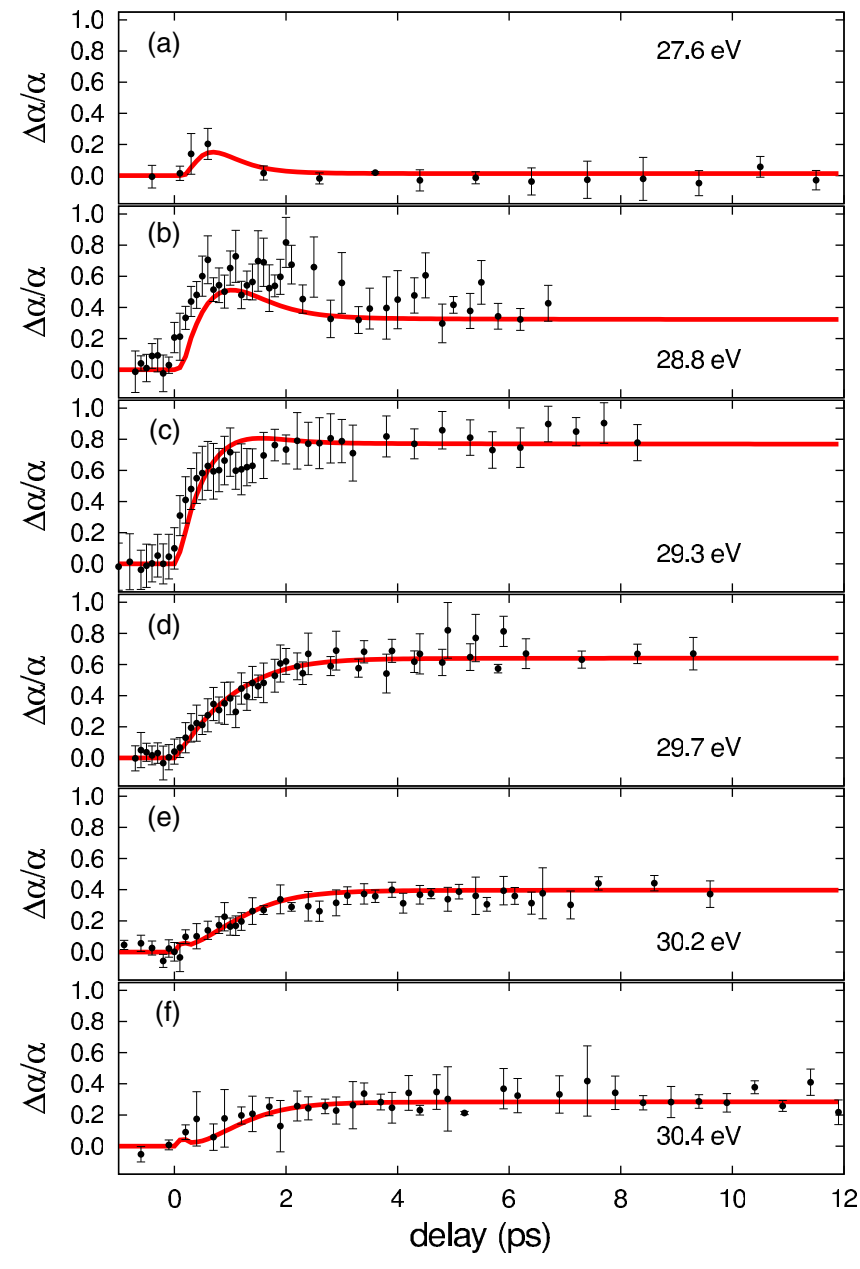

FIG. 2. Transient relative absorption changes $(\Delta \alpha / \alpha)$ at selected photon energies as a function of the delay between the optical laser pump and the FEL probe. The black circles are experimental data obtained with a pump fluence of (a) $60 \mathrm{~mJ} \mathrm{~cm}^{-2}$ and (b)-(f) $45 \mathrm{~mJ} \mathrm{~cm}^{-2}$. The red curves are calculated using Eq. (4) for a pump fluence of $45 \mathrm{~mJ} \mathrm{~cm}^{-2}$.

heated $\mathrm{Si}$ [15] and the $K$ edge of melted Ge [16]. These shifts of the $K$ and $L_{1}$ absorption edges in semiconductors are driven by the drastic increase in available electronic $p$-symmetric states across the Fermi level, following the band-gap collapse when the sample becomes metallic. Photoabsorption from the $s(K$ or $L_{1}$ edge) or $d$-symmetric core states $\left(M_{4,5}\right.$ edge as discussed here) directly probe this $p$ density of states ( $p$-DOS) according to the dipole selection rule.

In Fig. 2 we show the relative absorption variation $(\Delta \alpha / \alpha)$ for a set of selected probe photon energies $(E)$ in a time window of about $12 \mathrm{ps}$. Changes are observed to follow a different time profile at each energy. We assume the location of the Fermi level at the energy of $E_{0}=29.5 \mathrm{eV}$ close to the onset of the $M_{4,5}$ edge. In Fig. 2(a), $E=27.6 \mathrm{eV}$, dynamics in a region of the valence band significantly below the Fermi level are probed. Figures 2(b)-2(f) show the probe tuned just below $(E=28.8,29.3 \mathrm{eV})$ as well as above the Fermi level $(E=$ 29.7,30.2,30.4 eV). The observed change in absorption far below the Fermi level [Fig. 2(a)] recovers after 2 ps. Additional measurements show that the amplitude of this fluctuation 


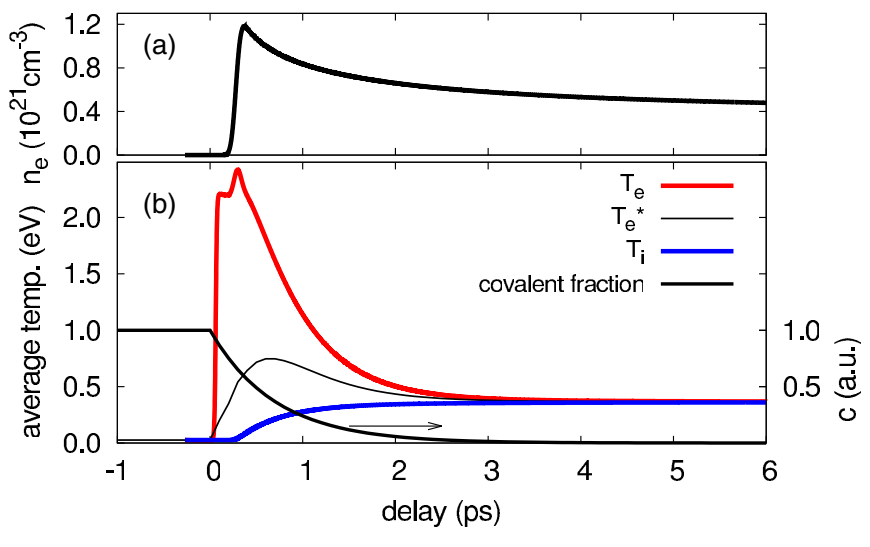

FIG. 3. Time-dependent quantities calculated for Ge by the nTTM [17] using a pump pulse with a wavelength of $780 \mathrm{~nm}$, a duration of $90 \mathrm{fs}$, and a fluence of $45 \mathrm{~mJ} \mathrm{~cm}^{-2}$. (a) Average free-electron density $\left(n_{e}\right)$; initial room-temperature value: $n_{e}^{0}=2.33 \times 10^{13} \mathrm{~cm}^{-3}$ [17]. (b) Average free electron and ion temperatures $\left(T_{e}\right.$ and $T_{i}$, respectively). $T_{e}^{*}$ represents the effective electron temperature [Eq. (2)]. $c(t)$ provides an estimate of the covalently bonded fraction in the sample.

is proportional to the pump fluence (see the Supplemental Material [17]). We connect this change directly with the redistribution of electrons according to their temperature in this case rather unaffected by changes in the electronic structure as a result of atomic rearrangements. On the contrary, the relative absorption variation right across the Fermi level [Figs. 2(b)$2(\mathrm{f})]$ is persistent and reaches the maximum amplitude already at lower pump fluences $\left(45 \mathrm{~mJ} \mathrm{~cm}^{-2}\right)$. From tabulated heat capacity and the latent heat of the melting of Ge, we expect that a few tens of $\mathrm{mJ} / \mathrm{cm}^{2}$ are sufficient to melt the sample volume in this experiment. Therefore, we associate the changes in Figs. 2(b)-2(f) with the melting and metallization dynamics of the Ge sample.

Time-dependent density functional theory (TD-DFT) $a b$ initio codes are ideally suitable to connect the observed time- and energy-resolved absorption data set as shown in Fig. 2 with the underlying microscopic evolution. However, TD-DFT simulations capable to effectively predict the time evolution of laser-excited nonequilibrium high energy density solids are extremely complex and require dedicated access to numerical calculation facilities. Therefore, we have developed a simplified model that combines numerical and analytical calculations. The used numerical code is based on a densitydependent two-temperature model (nTTM) developed for $\mathrm{Si}$ by Rämer et al. [18] and adapted to the Ge case. Such a MATLAB code is included in the Supplemental Material [17]. The numerical calculations have been used to model the temporal dependence of the electron and lattice temperatures as a function of the depth in the Ge sample. Results averaged over the sample thickness for a pump fluence of $45 \mathrm{~mJ} \mathrm{~cm}^{-2}$ are reported in Fig. 3(b) showing that the maximum average electron temperature of about $2.5 \mathrm{eV}$ is reached within about $300 \mathrm{fs}$, whereas the thermalization between electrons and ions occurs after 3 to $4 \mathrm{ps}$ at a temperature of about $0.4 \mathrm{eV}$.

The nTTM, however, assumes a fixed density of states and does not account for the ultrafast melting processes that drastically alter the electronic and atomic structures in a few

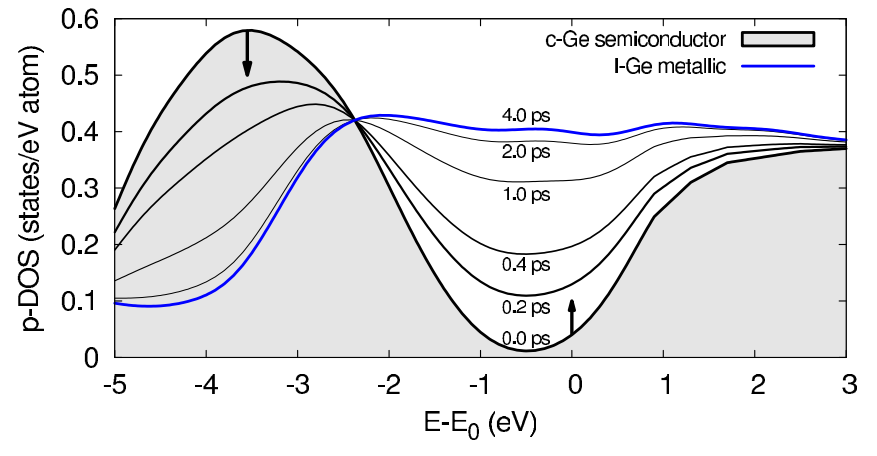

FIG. 4. Time evolution of the function $g(E, t)$ as defined in Eq. (1) in the spectral region across the Fermi level. $g(E, t)$ describes the average $p$-DOS in the Ge sample after exposure to an intense laser pulse.

hundreds of femtoseconds $[19,20]$. To include DOS changes, we combine the nTTM with the two-fluid model (TFM) [21]. With the TFM, we describe hot disordered Ge as a mixture of covalent (crystalline) and metallic (molten) fractions (c and $1-c$ ) [22]. Under equilibrium, the ratio between the two fractions is a function of pressure and temperature. In our nonequilibrium case though, we assume that the covalent fraction $c$ does not explicitly depend on those thermodynamic variables but solely on time: $c=c(t)=\exp \left(-t / \tau_{c}\right)$, whereas we model the melting dynamics with an exponential function, where $\tau_{c}$ is a model parameter. For the current data set, the model yields $\tau_{c}=0.7 \pm 0.3$ ps [Fig. 3(b)]. On the basis of the two-fluid description, the average $p$-DOS of nonequilibrium hot $\mathrm{Ge}[g(E, t)]$ is obtained as a linear combination of the $p$-DOS of a tetrahedrally coordinated covalent $\mathrm{Ge}$ and the $p$-DOS of the denser liquid metallic Ge,

$$
g(E, t)=c(t) g_{\mathrm{cov}}(E)+[1-c(t)] g_{\mathrm{met}}(E) .
$$

The functions $g_{\text {cov }}(E)$ and $g_{\text {met }}(E)$ have been obtained from the calculated $p$-DOS of crystalline and liquid Ge [23], slightly broadened in energy to account for atomic disorder of the microcrystalline Ge sample foil [17]. The obtained $g(E, t)$ distributions are presented in Fig. 4 for selected pump-probe delays, assuming a pump fluence of $45 \mathrm{~mJ} \mathrm{~cm}^{-2}$.

We further use the model to estimate the effective average electron temperature $T_{e}^{*}$. Indeed, prior to the complete metallization and melting of the sample, only a fraction of valence electrons can be considered as free and is excited to very high temperatures. According to the nTTM, within 100-200 fs after excitation, those free electrons amount to only about $1 \%$ of the valence electrons [Fig. 3(a)] but contain the entire amount of absorbed optical energy (about $2.5 \mathrm{eV} /$ electron). For later times, the fraction of free electrons increases rapidly as a result of the metallization, which we model with the exponential $c(t)$ function. Previous x-ray-diffraction studies have revealed the melting of about $40-50 \mathrm{~nm}$ of a Ge crystal by an $800-\mathrm{nm}$ laser to occur within a few hundred femtoseconds [7]. We thus assume that ultrafast melting occurs only in a fraction of the sample thickness $(m \simeq 0.6$ ), located below the surface where the hot free electrons are concentrated. Since the full sample volume is probed by absorption spectroscopy, this type of probe always averages over different regions of the sample. The 
effective electron temperature $T_{e}^{*}(t)$ [Fig. 3(b)] connected with the probed Fermi-Dirac distribution is empirically determined by using the functions $T_{i}(t)$ and $T_{e}(t)$ from the nTTM and the function $c(t)$ derived from the TFM,

$$
T_{e}^{*}(t)=(1-m) T_{i}(t)+m\left\{c(t) T_{i}(t)+[1-c(t)] T_{e}(t)\right\} .
$$

We consider the measured absorbance $\alpha(E, t)$ as the sum of a time-dependent part $\alpha_{3 d}(E, t)$ ascribed to the resonant absorption of $3 d$ electrons and a time-invariant part $\alpha_{b k g}(E)$ given by the nonresonant absorption of more weakly bound valence electrons. Since we work with comparably weak radiation fields, we neglect possible field-driven changes to both the atomic potential and the core-level binding energies. Furthermore, we neglect additional effects from electron-hole excitations, such as an energy shift or broadening of the valence and conduction bands that have been found to marginally alter the absorption across the Ge $M_{4,5}$ edge $\Delta \alpha / \alpha \lesssim 5 \%$ [10]. We thus determine the absorption coefficient at the $M_{4,5}$ edge via Fermi's golden rule [24], yielding,

$$
\alpha(E, t) \simeq A \rho(E, t)+\alpha_{b k g}(E),
$$

where $A$ is a scaling factor proportional to the squared matrix element $\left|\left\langle\Phi_{i}|\vec{e} \cdot \vec{r}| \Phi_{f}\right\rangle\right|^{2}$ and $\rho(E, t)$ represents the density of empty $p$-electronic states.

Within the above assumptions, the observed ultrafast changes in the sample absorbance are mostly driven by changes in $\rho(E, t)$ across the Fermi level. The function $\rho(E, t)$ is obtained as the product between the distribution function $\phi\left(E, T_{e}^{*}(t)\right)=1-f\left(E, T_{e}^{*}(t)\right)$, where $f$ is the Fermi-Dirac distribution and the $p$-DOS of $\mathrm{Ge}$ is given by the function $g(E, t)$ [Eq. (1)]. Therefore, we describe the relative variation of absorption for each delay by

$$
\frac{\Delta \alpha}{\alpha}(E, t) \simeq \frac{m A\left(\phi_{0} \Delta g+g_{0} \Delta \phi+\Delta g \Delta \phi\right)}{\alpha_{0}(E)} .
$$

where functions $\Delta g(E, t)$ and $\Delta \phi\left(E, T_{e}^{*}(t)\right)$ are absolute variations between time $t$ and time $t=0$ before excitation at a given photon energy and $g_{0}=g(E, 0), \phi_{0}=\phi\left(E, T_{e}^{*}(0)\right)$. The scale factor $A$ is determined to $A=4.1 \mathrm{eV}$ by comparing the experimental spectrum with the spectrum modeled by Eq. (3) for $t=0$. With the set of Eqs. (1)-(4), we completely model the experimental absorption data. In Fig. 2, the red solid curve presents the results from Eq. (4) for selected photon energies and for delays of $-1<t<12$ ps.

\section{CONCLUSIONS}

The agreement between the experimental data and the model is very good across the whole explored spectral and temporal ranges (Fig. 2). Therefore, we conclude that our approach using a limited number of free parameters and including several assumptions is justified and the following conclusions can be drawn.

First, the huge ultrafast variation of the Ge absorption coefficient across the $M_{4,5}$ edge ( $\left.\Delta \alpha / \alpha \approx 20-80 \%\right)$ triggered by an intense optical pump is a result of: (a) the redistribution of electrons according to an extremely high electron temperature and (b) the rearrangement of the electronic structure across the Fermi level associated with the band-gap collapse and the increase in available electronic $p$ states. Additional transient effects on $\Delta \alpha / \alpha$ driven by carrier dynamics [10] can be neglected under HED conditions. Under weak-field conditions as used here, the core electrons play a negligible role in the ultrafast absorption changes, and the matrix elements in the Fermi golden rule can be treated as invariant in both time and energy. This radically simplifies the simulations.

Second, the average electronic structure of HED Ge under nonequilibrium conditions can be effectively described as a time-dependent mixture of two well-defined electronic structures. The first is characterized by an energy gap at the Fermi level, typical of the tetrahedral atomic arrangement of covalent crystalline Ge. The second exhibits a constant high density of states across the Fermi level, typical of the denser atomic structure of metallic liquid Ge. The covalent fraction is still prevalent ( $c>0.8$, Fig. 3) even $300 \mathrm{fs}$ after pump exposure. This leads to a only partially filled $p$-DOS gap across the Fermi level (Fig. 4). On the other hand, $x$ ray-diffraction measurements [7] indicate that after $300 \mathrm{fs} \mathrm{Ge}$ is completely liquid. This is not contradictory to our results since $\mathrm{x}$-ray diffraction is sensitive to the long-range atomic order whereas absorption spectroscopy is sensitive to the local atomic arrangement. Merging both observations, we are led to believe that within 300 fs (i.e., prior to phonon heating), a large fraction of crystalline Ge undergoes nonthermal melting into a highly disordered phase still characterized by an energy gap (Fig. 4) and tetrahedral local coordination but devoid of a long-range-ordered structure. We call this intermediate phase the LDL. A tetrahedral phase, which is the stable configuration in crystalline $\mathrm{Ge}$ at room temperature, persists for about 1 ps above the melting point in a superheated transient regime. Interestingly, this scenario is validated by numerical simulations [25] that describe a laser-heated Si slab as a mixture of liquid nanometric droplets embedded in a superheated diamondlike lattice up to a depth of $100 \mathrm{~nm}$. The liquid fraction gradually increases through homogeneous nucleation. A process that is almost completed after a few picoseconds. Due to the structural and chemical analogy, the simulations can be considered representative for $\mathrm{Ge}$ as well and the simulated behavior agrees with the observations in this experiment. We conclude that the laser-heated HED Ge sample could possibly undergo two phase transitions. The first one, from crystalline to LDL Ge, is induced by nonthermal melting and occurs within 300 fs under isochoric conditions. The second one, from LDL to a stable phase compatible with a high-density-liquid Ge, results from a subsequent thermal evolution on the picosecond time scale under superheating conditions [26]. The rapidity of this melting mechanism could be ascribed to the supersonic melting front propagation typical of homogeneous nucleation [25].

Finally, we emphasize that nTTM simulations (Fig. 3) have been performed using parameters found in the literature for equilibrium Ge [17]. The only exception is the lattice heatcapacity $C_{p h}$. Indeed, the $C_{p h}$ value used for laser-heated Ge in numerical calculations $[27,28]$ cannot account for the high lattice temperature $(\sim 0.4 \mathrm{eV}, \sim 4850 \mathrm{~K})$ upon electron-lattice thermalization observed in our experiment [Fig. 3(b)]. In order to obtain an acceptable agreement between our experimental data and the sample temperature calculated by the nTTM, we need to use about $C_{p h}=0.4 \pm 0.1 \mathrm{~J} \mathrm{~cm}^{-3} \mathrm{~K}^{-1}$ [17], i.e., about $1 / 4$ of the literature value for equilibrium $\mathrm{Ge}$ at room 
temperature [27,28]. A similar observation has been made recently for $\mathrm{Cu}$ under $\mathrm{HED}$ conditions: Also there a notable contraction of the lattice heat capacity was observed [12]. This effect, potentially originating from an anomalous atomic bonding change upon HED conditions, needs further experimental investigation and should be considered in future theoretical calculations on laser-heated Ge. We can hypothesize that anomalous values of the lattice and electrons heat capacities $\left(C_{p h}\right.$ [12] and $C_{e}$ [29]) could be a characteristic feature of the warm dense matter regime.

In conclusion, the research presented in this paper shows the potential of time-resolved EUV absorption spectroscopy for investigating materials under nonequilibrium and HED conditions. A simulation framework has been presented for the interpretation of the experimental results. It was found to describe the main electronic and structural phenomena occurring in HED Ge driven out of equilibrium and subsequently relaxed at the margin of the warm dense matter region of the phase diagram. The proposed modeling can be extended to other materials, such as diamond, graphite, and silicon. This paper provides an alternative and valuable experimental benchmark that, we hope, will stimulate further discussions and theoretical elaborations.

\section{ACKNOWLEDGMENTS}

A. Giglia of the BEAR beamline is acknowledged for his assistance during XAS measurements at the Elettra synchrotron. The FERMI commissioning team is acknowledged for its invaluable technical assistance during FEL experiments.
[1] V. E. Fortov, Extreme States of Matter, 2nd ed., Springer Series in Materials Science Vol. 216 (Springer International Publishing, Cham, Switzerland, 2016).

[2] S. K. Sundaram and E. Mazur, Nat. Mater. 1, 217 (2002).

[3] B. Rethfeld, D. S. Ivanov, M. E. Garcia, and S. I. Anisimov, J. Phys. D 50, 193001 (2017).

[4] Frontiers and Challenges in Warm Dense Matter, edited by F. Graziani, M. P. Desjarlais, R. Redmer, and S. B. Trickey, Lecture Notes in Computational Science and Engineering (Springer International Publishing, Cham, Switzerland, 2014).

[5] R. W. Lee, S. J. Moon, H.-K. Chung, W. Rozmus, H. A. Baldis, G. Gregori, R. C. Cauble, O. L. Landen, J. S. Wark, A. Ng, S. J. Rose, C. L. Lewis, D. Riley, J.-C. Gauthier, and P. Audebert, J. Opt. Soc. Am. B 20, 770 (2003).

[6] C. W. Siders, A. Cavalleri, K. Sokolowski-Tinten, C. Tóth, T. Guo, M. Kammler, M. Horn von Hoegen, K. R. Wilson, D. von der Linde, and C. P. J. Barty, Science 286, 1340 (1999).

[7] K. Sokolowski-Tinten, C. Blome, C. Dietrich, A. Tarasevitch, M. H. von Hoegen, D. von der Linde, A. Cavalleri, J. Squier, and M. Kammler, Phys. Rev. Lett. 87, 225701 (2001).

[8] K. Sokolowski-Tinten and D. von der Linde, J. Phys.: Condens. Matter 16, R1517 (2004).

[9] M. Beye, F. Sorgenfrei, W. F. Schlotter, W. Wurth, and A. Foehlisch, Proc. Natl. Acad. Sci. USA 107, 16772 (2010).

[10] M. Zürch, H.-T. Chang, L. J. Borja, P. M. Kraus, S. K. Cushing, A. Gandman, C. J. Kaplan, M. H. Oh, J. S. Prell, D. Prendergast, C. D. Pemmaraju, D. M. Neumark, and S. R. Leone, Nat. Commun. 8, 15734 (2017).

[11] B. I. Cho, K. Engelhorn, A. A. Correa, T. Ogitsu, C. P. Weber, H. J. Lee, J. Feng, P. A. Ni, Y. Ping, A. J. Nelson, D. Prendergast, R. W. Lee, R. W. Falcone, and P. A. Heimann, Phys. Rev. Lett. 106, 167601 (2011).

[12] B. I. Cho, T. Ogitsu, K. Engelhorn, A. A. Correa, Y. Ping, J. W. Lee, L. J. Bae, D. Prendergast, R. W. Falcone, and P. A. Heimann, Sci. Rep. 6, 18843 (2016).

[13] M. B. Danailov, F. Bencivenga, F. Capotondi, F. Casolari, P. Cinquegrana, A. Demidovich, E. Giangrisostomi, M. P. Kiskinova, G. Kurdi, M. Manfredda, C. Masciovecchio, R. Mincigrucci, I. P. Nikolov, E. Pedersoli, E. Principi, and P. Sigalotti, Opt. Express 22, 12869 (2014).
[14] C. Masciovecchio, A. Battistoni, E. Giangrisostomi, F. Bencivenga, E. Principi, R. Mincigrucci, R. Cucini, A. Gessini, F. D’Amico, R. Borghes, M. Prica, V. Chenda, M. Scarcia, G. Gaio, G. Kurdi, A. Demidovich, M. B. Danailov, A. Di Cicco, A. Filipponi, R. Gunnella, K. Hatada, N. Mahne, L. Raimondi, C. Svetina, R. Godnig, A. Abrami, and M. Zangrando, J. Synchrotron Radiat. 22, 553 (2015).

[15] S. L. Johnson, P. A. Heimann, A. M. Lindenberg, H. O. Jeschke, M. E. Garcia, Z. Chang, R. W. Lee, J. J. Rehr, and R. W. Falcone, Phys. Rev. Lett. 91, 157403 (2003).

[16] A. Filipponi, M. Borowski, P. W. Loeffen, S. D. Panfilis, A. D. Cicco, F. Sperandini, M. Minicucci, and M. Giorgetti, J. Phys.: Condens. Matter 10, 235 (1998).

[17] See Supplemental Material at http://link.aps.org/supplemental/ 10.1103/PhysRevB.97.174107 for additional data analysis details.

[18] A. Rämer, O. Osmani, and B. Rethfeld, J. Appl. Phys. 116, 053508 (2014).

[19] P. Stampfli and K. H. Bennemann, Phys. Rev. B 49, 7299 (1994).

[20] K. Sokolowski-Tinten, J. Bialkowski, and D. von der Linde, Phys. Rev. B 51, 14186 (1995).

[21] L. I. Aptekar, Sov. Phys. Dokl. 24, 993 (1979).

[22] E. Principi, A. Di Cicco, F. Decremps, A. Polian, S. De Panfilis, and A. Filipponi, Phys. Rev. B 69, 201201 (2004).

[23] W. Jank and J. Hafner, Europhys. Lett. 7, 623 (1988).

[24] F. D. Groot and A. Kotani, Core Level Spectroscopy of Solids (CRC, Boca Raton, FL, 2008).

[25] V. P. Lipp, B. Rethfeld, M. E. Garcia, and D. S. Ivanov, Phys. Rev. B 90, 245306 (2014).

[26] B. Rethfeld, K. Sokolowski-Tinten, D. von der Linde, and S. I. Anisimov, Phys. Rev. B 65, 092103 (2002).

[27] J. Chen, D. Tzou, and J. Beraun, Int. J. Heat Mass Transfer 48, 501 (2005).

[28] F. Zhang, S. Li, A. Chen, Y. Jiang, S. Li, and M. Jin, High Power Laser Sci. Eng. 4, e12 (2016).

[29] B. Holst, V. Recoules, S. Mazevet, M. Torrent, A. Ng, Z. Chen, S. E. Kirkwood, V. Sametoglu, M. Reid, and Y. Y. Tsui, Phys. Rev. B 90, 035121 (2014). 